\title{
Microwave Kill Root Nematodes Based on the Arduino Automatic Control
}

\section{System}

\author{
Sheng Liu', Aisheng $\mathrm{Ma}^{2}$ and Xiumei Zhang ${ }^{3}$ \\ ${ }^{1}$ Weifang Science and Technology University, Shouguang, Shandong, 262700, China; \\ ${ }^{2}$ Weifang Science and Technology University, Shouguang, Shandong, 262700, China; \\ ${ }^{3}$ Weifang Science and Technology University, Shouguang, Shandong, 262700, China; \\ 1EE-mail:2948359382@qq.com,2E-mail:sg_xiaoma@qq.com, ${ }^{3}$ E-mail:582003674@qq.com.
}

Keywords: Pulse Microwave; The Microwave Oven; The Arduino; The Temperature Sensor; Silicon Controlled Rectifier

\begin{abstract}
For vegetable planting industry, root nematode is a kind of stubborn disease and insect pest. In order to improve the effect of kill root nematodes, the researchers use arduino as the core controller, use the touch switch and a temperature sensor through the sensor feedback signal, then change the pulse duty ratio control by thyristor temperature, and achieve the optimum insecticidal effect. Finally, the researchers conclude that with good temperature control, the microwave power is adjustable, the man-machine dialogue effect is good, the operation simple easy to master, and the automation level is high.
\end{abstract}

\section{Introduction}

Root nematodes become stubborn diseases in the growing of Chinese chives and cucumber, will cause the vegetable production as well as the "dirty" businessman will use dark medicine insecticidal caused by food safety hidden trouble. Microwave is a kind of can make the material in the inland waters molecules to vibrate and generate heat, therefore, we already use microwave oven to heat food in the our kitchen, and restaurant, the hotel has to be used for drying, sterilization equipment, the effect is obvious.

Currently infrared temperature detection device is used in the microwave oven device, and the sensors of the device are stable. As a result of microwave heating from the inside out, at this time of the infrared temperature sensor, temperature is only the surface temperature collected and the internal temperature of the heated material difference is very big. At the same time, now the microwave equipment for microwave magnetron is realized by using a timer to control, but this method reduces the life of a microwave magnetron, and also has impacts on the control system.

Based on the above problems, after studying, this paper put forward a new type of automatic control system of microwave kill root nematodes. This design uses the arduino as the control core, DS18B20 temperature acquisition module for the temperature control unit for soil temperature detection, closed-loop control to realize interpolation and interpolation signal transmission to the SCR to magnetron microwave output control. 


\section{Microwave Kill Root Nematode Composition of the System}

Microwave kill root nematode system main structure is shown in Fig. 1

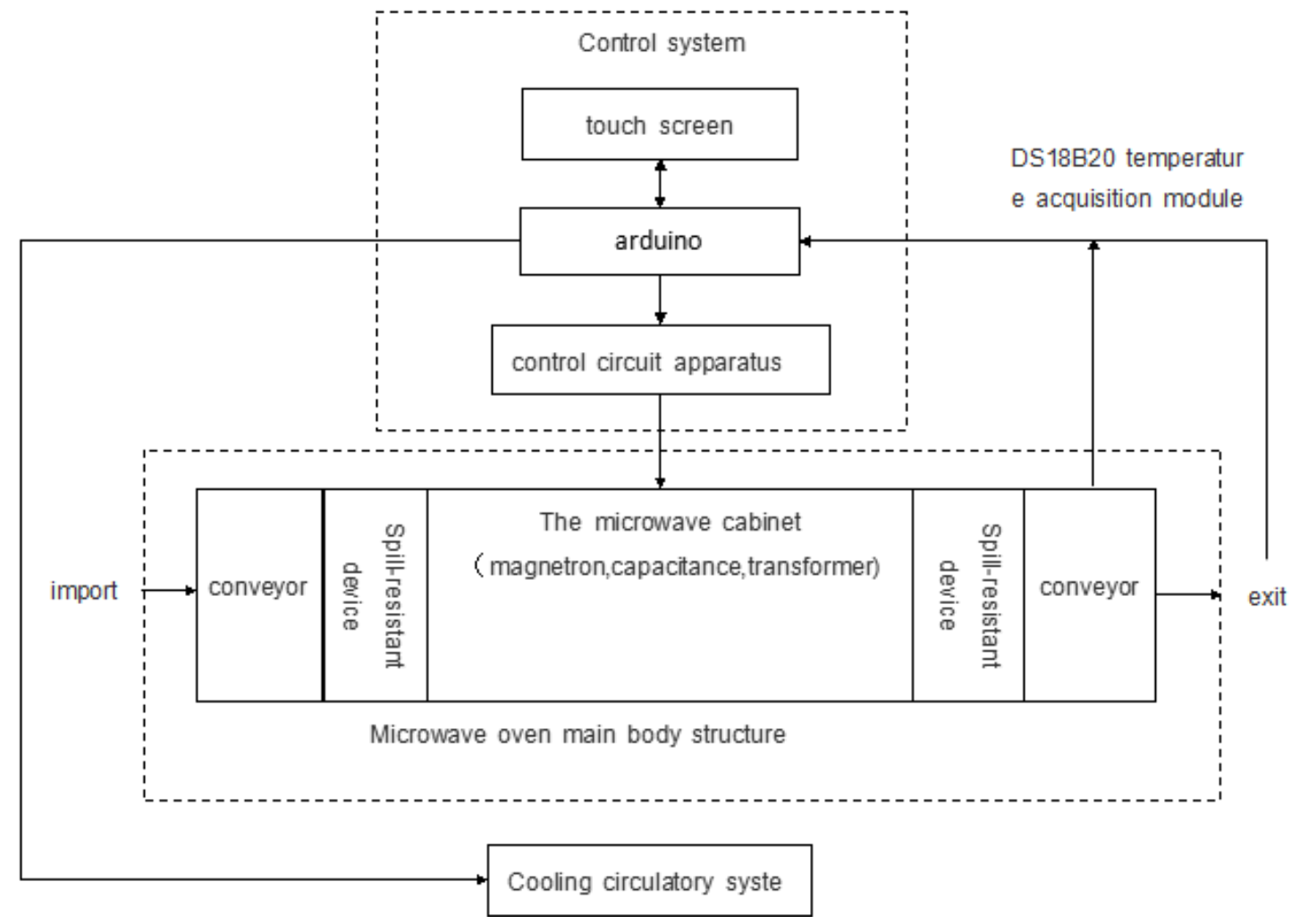

1) Magnetron and Resonant cavity and, the Funnel Type Structure

2) The rectangular frame installation of 12 groups of magnetron pulse current of capacitor and transformer and support. Type a panasonic m210-2 M1, maximum output power 900 w.Capacitor using the treasure chang $2100 \mathrm{v} 1 \mathrm{u}$ f microwave special capacitance, 12 groups of transformer the GAL - 700 - e - 1 s.36 sets of SCR control on rectangular frame is divided into three groups of 12 groups of magnetron.

3) This system uses the $12 \mathrm{v}$ supply to the transformer output for power supply way of wind cold short of 12 groups of fans.

4) The control of this system adopts the arduino and touch keys as core controller to realize automatic control.

5) Temperature detection and feedback system use the DS18B20 temperature acquisition module, and the test illuminates the internal temperature of the soil, the closed-loop control to realize interpolation

6) The microwave will cause certain damage to human body, so the funnel type stainless steel sheet is adopted to prevent overflow, microwave scattering and in use process, composed of overburden bucket cavity opening toward the ground soil.

\section{Microwave Kill Root Nematodes of Electrical Control System}

The control system of microwave kill unit root nematode mainly consists of the arduino and relevant input and output unit, temperature display, SCR, temperature sensor and controlled object

Microwave kill root nematode systemhardware design. Arduino port circuit design: 
According to the control requirements of the system, this paper requires eight digital output port and four analog input ports and three analog output ports. In order to meet this requirement, the researchers choose the Arduino UNO platform, and this platform is the core of ATmega328 processing chip havinga total of 14 digital input/output: 6 PWM output port road, road analog input port, all the way ICSP header, a $16 \mathrm{MHZ}$ crystal oscillator, a power switch, a USB interface and a reset button. In AREF, two pins are added: SCL and SDA, support the I2C interface; increase IOREF and reserve a pin, control panel can be compatible with $5 \mathrm{~V}$ and $3.3 \mathrm{~V}$ supply voltage.

Microwave kill root nematode circuit design of the system:

Choose the heating unit of intelligent thyristor control insecticidal machine, this system uses MJYS - JL - 450 type intelligent thyristor, and thyristor module of the input signal is $4 \mathrm{ma}-20 \mathrm{ma}$ current or voltage 0 to $5 \mathrm{v}$.

The system control method is as follows:

1) Use the arduino form PWM pulse signal timing control function, low elecrial level is the input signal with 4 ma thyristor, magnetron no microwave power is zero at this time; High electricity at ordinary times, silicon controlled rectifier input signal to $20 \mathrm{ma}$, microwave magnetron, at this time a single power $900 \mathrm{w}$.

2) Power regulation. Through the change of the PWM duty cycle of the signal, when the duty ratio is less than 1 , can activate the microwave magnetron output. So you can through the PWM pulse width modulation pulse rate and silicon controlled rectifier input signal to change the output power of microwave

Microwave kill root nematode temperature control circuit design of the system:

In the surrounding temperature, root nematodes reaches 50 degrees Celsius that can be effectively killed, so a lot of vegetable farmers plant cucumbers in susceptible root nematode vegetables stuffy tent measures to kill the root nematodes, which has achieved a certain effect. Therefore, the key to the core of this system is to control the temperature of the microwave insecticidal, in order to avoid high temperature kill good bacteria in the soil, and also avoid reaching 55 degrees Celsius temperature that cannot effectively kill root nematodes.

In the system of the temperature feedback collection unit, it adopts the digital temperature sensor DS18B20, the module will collect the temperature of the analog signal into digital signal, and the sensor includes a NTC thermometer element and a resistive touch wet element. DS18B20 temperature detection range is $-55 \sim+125{ }^{\circ} \mathrm{C}$ in $-10 \sim+85{ }^{\circ} \mathrm{C}$ with an accuracy of $+/-5{ }^{\circ} \mathrm{C}$. In each bucket cover, the bottom has a DS18B20, soil temperature signal collected and processed output PWM pulse signal, control thyristor module, the power magnetron is changed, and the temperature is controlled.

Microwave kill root ne matode system software design. This system adopts the aiduino as processing core, and the main program includes the main program, subprogram, temperature acquisition and practical program, microwave control instruction, fault alarm procedures, etc.

Microwave kill root nematode system temperature and pulse microwave control program:

The core of this system is the control for temperature and microwave power, the collection and processing of soil temperature DS18B20 temperature sensor. The core of this system is the control for temperature and microwave power, the collection and processing of soil temperature DS18B20 temperature sensor. This system has two kinds of manual and automatic operation mode. Automatic mode, the use of pid algorithm, and calculate the duty ratio, through the arduino central processing unit and output control signal, through intelligent thyristor adjust the duty ratio of pulse signal, 
change the power of microwave magnetron to for temperature control. When malfunction or insecticidal effect is not ideal the need for manual operation, the operator can be manually opened according to the actual temperature heating, namely in setting an interrupt program, through the manual button at the bottom of the screen to switch to the program change PMW duty ratio to change the role of the magnetron power.

This system program design flow chart is shown in Fig. 4.

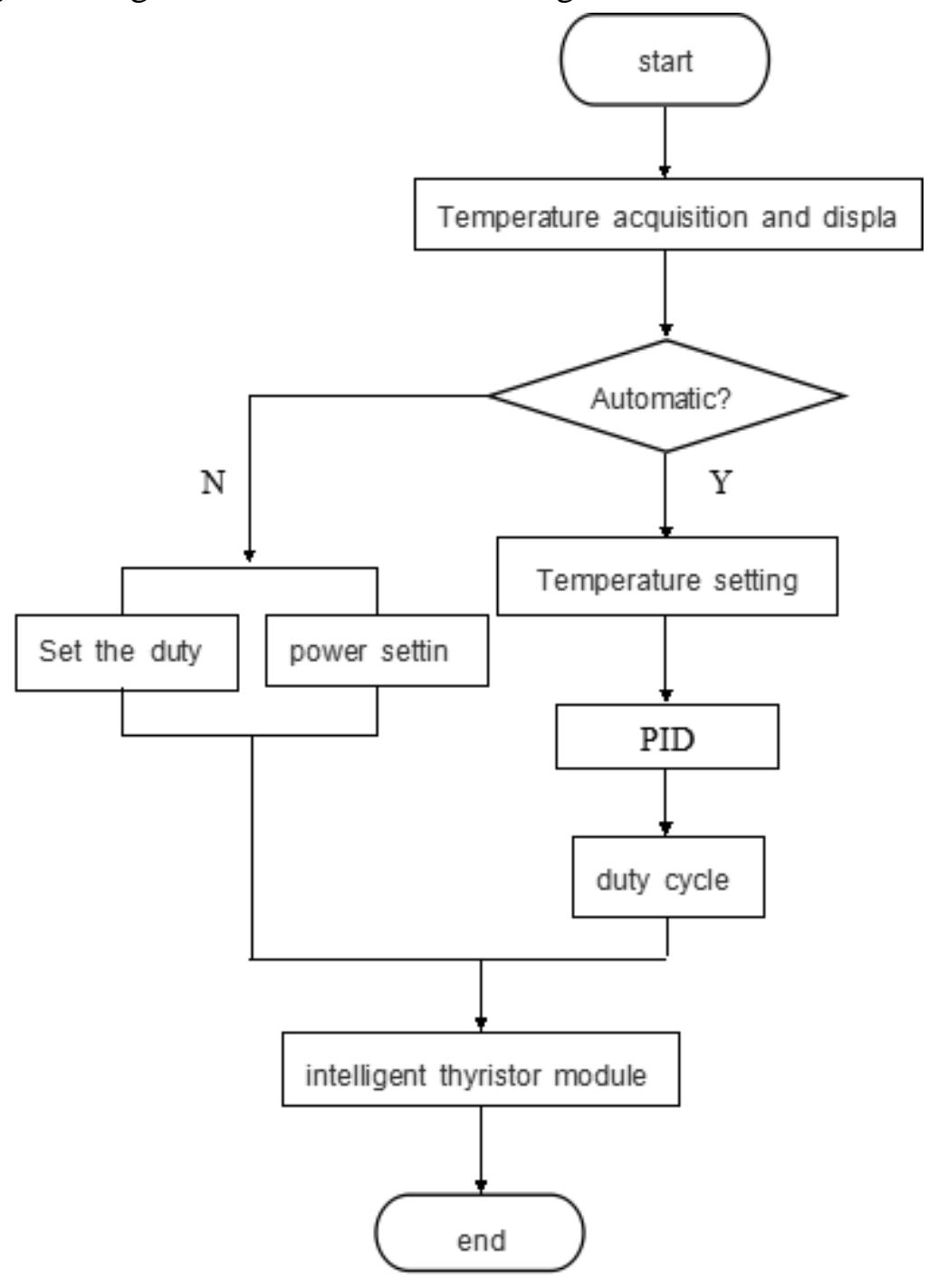

\section{Conclusions}

This system adopts the arduino as the core of the control unit, by controlling the output power of microwave pulse duty ratio control. And USES DS18B20 temperature sensor to collect signal and output control signal so that a complete output, execution, feedback, to perform a closed-loop control. Using this device can effectively kill the root nematodes, and reduce pollution

\section{References}

[1] Zhang Qiangwu, Tang Luxin, Chen Chaofeng, Fan Hangjun. Microcontrollers Embedded Systems. Beijing University of Aeronautics and Astronautics Press, 2001. School of Information Engineering, Guangdong University of Technology. 
[2] Song Hui,Wang Zhichan. Studies of the temperature control system based on single-chip microcomputer. Electronic production, 2014.2.

[3] Nie Shaowu,Huang Hanying, Hu Yuelai, Zhao Siming.Grain pulse microwave insecticidal machine based on PLC automatic control system Manufacturing Automation, 2014. The mechanical machinery industrial automation rese arch institute in Beijing.

[4] Zhang Zhiyong,Wei Yong,Chan Huiyong.A kind of digital thermometer based on single-chip microcomputer control design. Journal of Tianjin Agricultural College (11).

[5] Liu Yan. Effect of microwave irradiation effect on pests. Henan University of Technology, 2010.

[6]Wan Pingshi. Based on pulse width modulation experimental study of variable spraying technology. Heilongiiang August first land reclamation university in 2007.

[7]Cong Hongshou, Wu Duoxin, Yan Zhixin. ZK - 1 silicon controlled voltage regulator in the application of the furnace temperature control. The second session of the national annual meeting of the special power supply and components, 2002.

[8]Zhang Jinbao, ShaoMingxi, Huang Zongjing. A kind of neutralizing the longicorn larvae microwave insecticidal machine, Chinese patent,CN102487919A, 2012-6-3. 